THU-98/44

hep-th/9812082

\title{
Corrections to macroscopic supersymmetric black-hole entropy
}

\author{
Gabriel Lopes Cardoso ${ }^{1}$, Bernard de Wit ${ }^{1}$ and Thomas Mohaupt ${ }^{2}$ a \\ ${ }^{1}$ Institute for Theoretical Physics, Utrecht University, 3508 TA Utrecht, The Netherlands \\ ${ }^{2}$ Martin-Luther-Universität Halle-Wittenberg, Fachbereich Physik, D-06099 Halle, Germany
}

\begin{abstract}
We determine the corrections to the entropy of extremal black holes arising from terms quadratic in the Riemann tensor in $N=2, D=4$ supergravity theories. We follow Wald's proposal to modify the Bekenstein-Hawking area law. The new entropy formula, whose value only depends on the electric/magnetic charges, is expressed in terms of a single holomorphic function and is consistent with electric-magnetic duality. For string effective field theories arising from Calabi-Yau compactifications, our result for the entropy of a certain class of extremal black-hole solutions fully agrees with the counting of microstates performed some time ago by Maldacena, Strominger, Witten and by Vafa.
\end{abstract}

December 1998

\footnotetext{
${ }^{a}$ cardoso@phys.uu.nl

${ }^{b}$ bdewit@phys.uu.nl

${ }^{c}$ mohaupt@hera1.physik. uni-halle.de
} 
Some time ago the microscopic entropy was determined for certain black holes arising in CalabiYau compactifications of M-theory and type-IIA string theory [1, 2]. The results cannot be reconciled with the macroscopic entropy formulae based on an effective $N=2$ supergravity

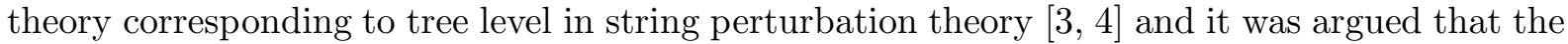
deviations arise from terms in the effective action proportional to the square of the Riemann tensor, with coefficients linearly related to the second Chern class of the Calabi-Yau manifold. The latter arise as one-loop corrections in string perturbation theory. At the microscopic level the deviations correspond to subleading corrections to the central charge of a two-dimensional conformal field theory and were already present in the results of [5]. In [1, 2] this conjectured correspondence between microscopic and macroscopic results was verified, based on certain assumptions (such as the constancy of the moduli), to first order in the coefficients of the $R^{2}$-terms. Subsequently an attempt was made to determine the macroscopic result in the context of a full $N=2$ supersymmetric effective field theory with $R^{2}$-terms, exploiting the fixed-point behaviour of the moduli fields and many features of special geometry [6]. Somewhat surprisingly, however, it was found that the first-order correction to the macroscopic entropy vanishes, although higher-order terms are indeed present.

In this note we return to this question and completely resolve the discrepancy. In doing so we are able to go beyond the specific cases discussed above and to treat a much larger variety of 4-dimensional $N=2$ supersymmetric black-hole solutions. A crucial ingredient of our work is that we depart from the Bekenstein-Hawking area formula and follow Wald's proposal for the entropy formula based on a Noether charge associated with an isometry evaluated at the corresponding Killing horizon [7]. Let us recall that the black-hole solutions that we consider are static, rotationally symmetric solitonic interpolations between two $N=2$ supersymmetric groundstates: flat Minkowski spacetime at spatial infinity and Bertotti-Robinson spacetime at the horizon [8]. Hence we can parametrize the spacetime line element in terms of two functions of the radius,

$$
d s^{2}=-e^{2 g(r)} d t^{2}+e^{2 f(r)}\left[d r^{2}+r^{2}\left(\sin ^{2} \theta d \phi^{2}+d \theta^{2}\right)\right],
$$

in so-called isotropic coordinates. The interpolating solution preserves $N=1$ supersymmetry so that we are dealing with a BPS configuration and the black hole is extremal. The residual supersymmetry is also responsible for the fixed-point behaviour of the moduli and forces them to take certain values depending on the electric/magnetic charges [3] (for a recent reference on the fixed-point behaviour, see [9]). Assuming fixed-point behaviour, the precise relation follows exclusively from electric-magnetic duality considerations [4]. By making use of these features, it remains to analyze the restrictions posed by full $N=2$ supersymmetry on a static and spherically symmetric geometry. Here it is important to realize that, in view of the fact that the action contains $R^{2}$-terms, one has to make optimal use of the existence of a full off-shell multiplet calculus for these theories [10]. This enables one to perform the analysis in a way that is independent of the complications of the action, although ultimately the full field equations are indeed satisfied for supersymmetric field configuration.

The multiplet calculus that we employ is based on the gauge symmetries of the superconformal algebra. The corresponding high degree of symmetry allows for the use of relatively small off-shell field representations. One is the Weyl multiplet, whose fields comprise the gauge fields corresponding to the superconformal symmetries and a few auxiliary fields. As we will demonstrate, 
it is possible to analyze the conditions for $N=2$ supersymmetry directly in this formulation, postponing a transition to Poincaré supergravity till the end. Poincaré supergravity theories are obtained by coupling additional superconformal multiplets containing Yang-Mills and matter fields to the Weyl multiplet. Under certain conditions the resulting superconformal theory then becomes gauge equivalent to a theory of Poincaré supergravity. Some of the additional multiplets are necessary to provide compensating fields and to overcome a deficit in degrees of freedom between the Weyl multiplet and the Poincaré supergravity multiplet. For instance, the graviphoton, represented by an abelian vector field in the Poincaré supergravity multiplet, is provided by an $N=2$ superconformal vector multiplet. However, as mentioned above, the main part of our analysis remains within the context of the full superconformal formulation. This implies in particular that all our results (including (11)) are subject to local scale transformations. Only towards the end we will convert to expressions that are scale invariant.

The superconformal algebra contains general-coordinate, local Lorentz, dilatation, special conformal, chiral SU(2) and $\mathrm{U}(1)$, supersymmetry $(Q)$ and special supersymmetry $(S)$ transformations. The gauge fields associated with general-coordinate transformations $\left(e_{\mu}^{a}\right)$, dilatations $\left(b_{\mu}\right)$, chiral symmetry $\left(\mathcal{V}_{\mu j}^{i}, A_{\mu}\right)$ and $Q$-supersymmetry $\left(\psi_{\mu}^{i}\right)$, are realized by independent fields. The remaining gauge fields of Lorentz $\left(\omega_{\mu}^{a b}\right)$, special conformal $\left(f_{\mu}^{a}\right)$ and $S$-supersymmetry transformations $\left(\phi_{\mu}^{i}\right)$ are dependent fields. They are composite objects, which depend in a complicated way on the independent fields [10]. The corresponding curvatures and covariant fields are contained in a tensor chiral multiplet, which comprises $24+24$ off-shell degrees of freedom; in addition to the independent superconformal gauge fields it contains three auxiliary fields: a Majorana spinor doublet $\chi^{i}$, a scalar $D$ and a selfdual Lorentz tensor $T_{a b i j}$ (where $i, j, \ldots$ are chiral $\mathrm{SU}(2)$ spinor indices) 1 . We summarize the transformation rules for some of the independent fields of the Weyl multiplet under $Q$ - and $S$-supersymmetry and under special conformal transformations, with parameters $\epsilon^{i}, \eta^{i}$ and $\Lambda_{\mathrm{K}}^{a}$, respectively,

$$
\begin{aligned}
\delta e_{\mu}{ }^{a}= & \bar{\epsilon}^{i} \gamma^{a} \psi_{\mu i}+\text { h.c. } \\
\delta \psi_{\mu}^{i}= & 2 \mathcal{D}_{\mu} \epsilon^{i}-\frac{1}{8} T^{a b i j} \gamma_{a b} \gamma_{\mu} \epsilon_{j}-\gamma_{\mu} \eta^{i}, \\
\delta b_{\mu}= & \frac{1}{2} \bar{\epsilon}^{i} \phi_{\mu i}-\frac{3}{4} \bar{\epsilon}^{i} \gamma_{\mu} \chi_{i}-\frac{1}{2} \bar{\eta}^{i} \psi_{\mu i}+\text { h.c. }+\Lambda_{\mathrm{K}}^{a} e_{\mu}^{a}, \\
\delta A_{\mu}= & \frac{1}{2} i \bar{\epsilon}^{i} \phi_{\mu i}+\frac{3}{4} i \bar{\epsilon}^{i} \gamma_{\mu} \chi_{i}+\frac{1}{2} i \bar{\eta}^{i} \psi_{\mu i}+\text { h.c. }, \\
\delta T_{a b}^{i j}= & 8 \bar{\epsilon}^{[i} R(Q)_{a b}^{j]}, \\
\delta \chi^{i}= & -\frac{1}{12} \gamma_{a b} \not D T^{a b i j} \epsilon_{j}+\frac{1}{6} R(\mathcal{V})_{a b}{ }_{j}^{i} \gamma^{a b} \epsilon^{j}-\frac{1}{3} i R(A)_{a b} \gamma^{a b} \epsilon^{i} \\
& +D \epsilon^{i}+\frac{1}{12} T_{a b}^{i j} \gamma^{a b} \eta_{j},
\end{aligned}
$$

where $\mathcal{D}_{\mu}$ are derivatives covariant with respect to Lorentz, dilatational, $\mathrm{U}(1)$ and $\mathrm{SU}(2)$ transformations, and $D_{\mu}$ are derivatives covariant with respect to all superconformal transformations. Throughout this paper we suppress terms of higher order in the fermions, as we will be dealing with a bosonic background. The quantities $R(Q)_{\mu \nu}^{i}, R(A)_{\mu \nu}$ and $R(\mathcal{V})_{\mu \nu}{ }^{i}{ }_{j}$ are supercovariant curvatures related to $Q$-supersymmetry, $\mathrm{U}(1)$ and $\mathrm{SU}(2)$ transformations.

Let us now turn to the abelian vector multiplets, labelled by an index $I=0,1, \ldots, n$. For each

\footnotetext{
${ }^{d}$ By an abuse of terminology, $T_{a b i j}$ is often called the graviphoton field strength. It is antisymmetric in both Lorentz indices $a, b$ and chiral $\mathrm{SU}(2)$ indices $i, j$. Its complex conjugate is the anti-selfdual field $T_{a b}^{i j}$. Our conventions are such that $\mathrm{SU}(2)$ indices are raised and lowered by complex conjugation. The SU(2) gauge field $\mathcal{V}_{\mu j}^{i}$ is antihermitean and traceless, i.e., $\mathcal{V}_{\mu j}^{i}+\mathcal{V}_{\mu j}{ }^{i}=\mathcal{V}_{\mu i}^{i}=0$.
} 
value of the index $I$, there are $8+8$ off-shell degrees of freedom, residing in a complex scalar $X^{I}$, a doublet of chiral fermions $\Omega_{i}^{I}$, a vector gauge field $W_{\mu}^{I}$, and a real $\mathrm{SU}(2)$ triplet of scalars $Y_{i j}^{I}$. Under $Q$ - and $S$-supersymmetry the fields $X^{I}$ and $\Omega_{i}^{I}$ transform as follows:

$$
\begin{aligned}
& \delta X^{I}=\bar{\epsilon}^{i} \Omega_{i}^{I}, \\
& \delta \Omega_{i}^{I}=2 \not D X^{I} \epsilon_{i}+\frac{1}{2} \varepsilon_{i j} \mathcal{F}_{\mu \nu}^{I-} \gamma^{\mu \nu} \epsilon^{j}+Y_{i j}^{I} \epsilon^{j}+2 X^{I} \eta_{i} .
\end{aligned}
$$

The quantities $\mathcal{F}_{\mu \nu}^{I}$ are related to the abelian field strengths by

$$
\mathcal{F}_{\mu \nu}^{I}=F_{\mu \nu}^{I}-\frac{1}{4}\left(\varepsilon_{i j} \bar{X}^{I} T_{\mu \nu}^{i j}+\text { h.c. }\right), \quad F_{\mu \nu}^{I}=2 \partial_{[\mu} W_{\nu]}^{I} .
$$

The covariant quantities of the vector multiplet constitute a reduced chiral multiplet. A general chiral multiplet comprises $16+16$ off-shell degrees of freedom and carries an arbitrary Weyl weight $w$ (corresponding to the Weyl weight of its lowest component). The covariant quantities of the vector multiplet may be obtained from a chiral multiplet with $w=1$ by the application of a set of reducibility conditions, one of which is the Bianchi identity. Similarly, the covariant quantities of the Weyl multiplet also constitute a reduced chiral multiplet, denoted by $W^{a b i j}$, whose lowest $\theta$ component is the tensor $T^{a b i j}$. From this multiplet one may form a scalar (unreduced) chiral multiplet $W^{2}=\left[W^{a b i j} \varepsilon_{i j}\right]^{2}$ which has Weyl and chiral weights $w=2$ and $c=-2$, respectively [11].

In the following, we will also allow for the presence of an arbitrary chiral background superfield [12], whose component fields will be indicated with a caret. Eventually this multiplet will be identified with $W^{2}$ in order to generate the $R^{2}$-terms in the action, but much of our analysis will not depend on its precise identification. We denote its bosonic component fields by $\hat{A}, \hat{B}_{i j}$, $\hat{F}_{a b}^{-}$and by $\hat{C}$. Here $\hat{A}$ and $\hat{C}$ denote complex scalar fields, appearing at the $\theta^{0}$ - and $\theta^{4}$-level of the chiral background superfield, respectively, while the symmetric complex $\mathrm{SU}(2)$ tensor $\hat{B}_{i j}$ and the anti-selfdual Lorentz tensor $\hat{F}_{a b}^{-}$reside at the $\theta^{2}$-level. The fermion fields at level $\theta$ and $\theta^{3}$ are denoted by $\hat{\Psi}_{i}$ and $\hat{\Lambda}_{i}$. Under $Q$ - and $S$-supersymmetry $\hat{A}$ and $\Psi_{i}$ transform as

$$
\begin{aligned}
\delta \hat{A} & =\bar{\epsilon}^{i} \hat{\Psi}_{i}, \\
\delta \hat{\Psi}_{i} & =2 \not D \hat{A} \epsilon_{i}+\frac{1}{2} \varepsilon_{i j} \hat{F}_{a b} \gamma^{a b} \epsilon^{j}+\hat{B}_{i j} \epsilon^{j}+2 w \hat{A} \eta_{i},
\end{aligned}
$$

where $w$ denotes the Weyl weight of the background superfield. In the presence of this chiral background superfield, the coupling of the abelian vector multiplets to the Weyl multiplet is encoded in a function $F\left(X^{I}, \hat{A}\right)$, which is holomorphic and homogenous of degree two,

$$
X^{I} F_{I}+w \hat{A} F_{\hat{A}}=2 F, \quad F_{I}=\partial_{X^{I}} F, \quad F_{\hat{A}}=\partial_{\hat{A}} F .
$$

We will employ this function in what follows, although we are not (yet) dealing directly with the action.

The field equations of the vector multiplets are subject to equivalence transformations corresponding to electric-magnetic duality, which will not involve the fields of the Weyl multiplet and of the chiral background. As is well-known, two complex $(2 n+2)$-component vectors can be defined which transform linearly under the $\mathrm{SP}(2 n+2 ; \mathbf{R})$ duality group, namely

$$
V=\left(\begin{array}{c}
X^{I} \\
F_{I}(X, \hat{A})
\end{array}\right) \quad \text { and } \quad\left(\begin{array}{c}
F_{\mu \nu}^{+I} \\
G_{\mu \nu I}^{+}
\end{array}\right) \text {. }
$$


The first vector has weights $w=1$ and $c=-1$, whereas the second one has zero Weyl and chiral weights. The field strengths $G_{\mu \nu I}^{ \pm}$are defined as follows:

$$
G_{\mu \nu I}^{+}=\mathcal{N}_{I J} F_{\mu \nu}^{+J}+\mathcal{O}_{\mu \nu I}^{+}, \quad G_{\mu \nu I}^{-}=\overline{\mathcal{N}}_{I J} F_{\mu \nu}^{-J}+\mathcal{O}_{\mu \nu I}^{-},
$$

where

$$
\mathcal{N}_{I J}=\bar{F}_{I J}, \quad \mathcal{O}_{\mu \nu I}^{+}=\frac{1}{4}\left(F_{I}-\bar{F}_{I J} X^{J}\right) T_{\mu \nu i j} \varepsilon^{i j}+\hat{F}_{\mu \nu}^{+} \bar{F}_{I \hat{A}}
$$

They appear in the field equations of the vector fields (in the presence of the background). Solving Bianchi identities, $\mathcal{D}^{a}\left(F^{-}-F^{+}\right)_{a b}^{I}=0$, and field equations, $\mathcal{D}^{a}\left(G^{-}-G^{+}\right)_{a b}^{I}=0$, in the static, spherically symmetric geometry (1) with the chiral background turned on, defines the magnetic/electric charges $\left(p^{I}, q_{J}\right)$, which therefore comprise a symplectic vector (note that we use tangent-space indices $a=0,1,2,3$ corresponding to $\mu=t, r, \phi, \theta)$,

$$
\begin{gathered}
F_{01}^{-I}-F_{01}^{+I}=i F_{23}^{I}=i \frac{\mathrm{e}^{-2 f(r)}}{r^{2}} p^{I}, \\
G_{01 I}^{-}-G_{01 I}^{+}=i G_{23 I}=i \frac{\mathrm{e}^{-2 f(r)}}{r^{2}} q_{I} .
\end{gathered}
$$

Assuming a fixed-point behaviour for the moduli at the horizon, the symplectic covariance implies that the symplectic vector $V$ must be proportional to the symplectic charge vector $\left(p^{I}, q_{J}\right)[6]$. This yields

$$
\bar{Z}\left(\begin{array}{c}
X^{I} \\
F_{J}
\end{array}\right)-Z\left(\begin{array}{c}
\bar{X}^{I} \\
\bar{F}_{J}
\end{array}\right)=i \mathrm{e}^{-\mathcal{K} / 2}\left(\begin{array}{c}
p^{I} \\
q_{J}
\end{array}\right), \quad \text { with } \quad Z=\mathrm{e}^{\mathcal{K} / 2}\left(p^{I} F_{I}-q_{I} X^{I}\right),
$$

where we introduced the symplectically invariant factor (with $w=2$ and $c=0$ ),

$$
\mathrm{e}^{-\mathcal{K}}=i\left[\bar{X}^{I} F_{I}(X, \hat{A})-\bar{F}_{I}(\bar{X}, \overline{\hat{A}}) X^{I}\right]
$$

which resembles (but is not equal to) the Kähler potential in special geometry. By supersymmetry, this factor is related to the spinor,

$$
\zeta_{i} \equiv-\left(\Omega_{i}^{I} \frac{\partial}{\partial X^{I}}+\hat{\Psi}_{i} \frac{\partial}{\partial \hat{A}}\right) \mathcal{K}=-i \mathrm{e}^{\mathcal{K}}\left[\left(\bar{F}_{I}-\bar{X}^{J} F_{I J}\right) \Omega_{i}^{I}-\bar{X}^{I} F_{I \hat{A}} \hat{\Psi}_{i}\right]
$$

It can be shown, using the results contained in [12], that $\zeta_{i}$ is also inert under symplectic reparametrizations. Under $Q$ - and $S$-supersymmetry $\zeta_{i}$ transforms as

$$
\begin{aligned}
\delta \zeta_{i}= & -2 i \mathrm{e}^{\mathcal{K}}\left(\bar{F}_{I} \not D X^{I}-\bar{X}^{I} \not D F_{I}\right) \epsilon_{i}-i \mathrm{e}^{\mathcal{K}}\left[\left(\bar{F}_{I}-\bar{X}^{J} F_{J I}\right) Y_{i j}^{I}-\bar{X}^{I} F_{I A} \hat{B}_{i j}\right] \epsilon^{j} \\
& -\frac{1}{2} i \varepsilon_{i j} \mathcal{F}_{a b}^{-} \gamma^{a b} \epsilon^{j}+2 \eta_{i},
\end{aligned}
$$

where we ignored higher-order fermionic terms. Here $\mathcal{F}_{a b}^{-}$denotes the following (tangent-space) anti-selfdual tensor,

$$
\mathcal{F}_{a b}^{-}=\mathrm{e}^{\mathcal{K}}\left(\bar{F}_{I} F_{a b}^{-I}-\bar{X}^{I} G_{a b I}^{-}\right)
$$


Having fixed the relation between the charges and the values of the moduli at the horizon, the next step is to investigate the possible horizon geometry by imposing full $N=2$ supersymmetry. Let us first observe that the spinor $\zeta_{i}$ transforms inhomogenously under $S$-supersymmetry and hence it can act as a compensator for this symmetry. This observation is relevant when constructing supersymmetric backgrounds, where one requires the $Q$-supersymmetry variations of the spinors to vanish modulo a uniform $S$-transformation. This can conveniently be done by considering $S$-invariant spinors, constructed by employing $\zeta_{i}$. Relevant examples of such spinors are $\Omega_{i}^{I}-X^{I} \zeta_{i}, \hat{\Psi}_{i}-w \hat{A} \zeta_{i}, \chi^{i}-\frac{1}{24} T_{a b}^{i j} \gamma^{a b} \zeta_{j}$ and $R(Q)_{a b}^{i}-\frac{1}{16} T^{c d i j} \gamma_{c d} \gamma_{a b} \zeta_{j}$. Requiring the vanishing of the $Q$-supersymmetry variations of these $S$-invariant fermionic fields leads to a number of stringent conditions on the background, as follows:

$$
\begin{aligned}
& \left(\partial_{\mu}-i \mathcal{A}_{\mu}\right)\left(\begin{array}{c}
\mathrm{e}^{\mathcal{K} / 2} X^{I} \\
\mathrm{e}^{\mathcal{K} / 2} F_{I}(X, \hat{A})
\end{array}\right)=\left(\partial_{\mu}-i w \mathcal{A}_{\mu}\right)\left(\mathrm{e}^{w \mathcal{K} / 2} \hat{A}\right)=0, \\
& \mathcal{F}_{a b}^{-I}=-i X^{I} \mathcal{F}_{a b}^{-}, \quad X^{I} \hat{F}_{a b}^{-}=w \hat{A} \mathcal{F}_{a b}^{-I}, \\
& \mathcal{D}_{c} T_{a b}^{i j}=i \mathrm{e}^{\mathcal{K}}\left(\bar{X}^{J} \mathcal{D}_{d} F_{J}-\bar{F}_{J} \mathcal{D}_{d} X^{J}\right)\left(\delta_{c}^{d} T_{a b}^{i j}-2 \delta_{[a}^{d} T_{b] c}^{i j}+2 \delta_{c[a} T_{b]}^{i j d}\right),
\end{aligned}
$$

where $\mathcal{A}_{\mu}$ denotes a Kähler connection which must be flat,

$$
\mathcal{A}_{\mu}=\frac{1}{2} \mathrm{e}^{\mathcal{K}}\left(\bar{X}^{J} \stackrel{\leftrightarrow}{\partial}_{\mu} F_{J}-\bar{F}_{J} \overleftrightarrow{\partial}_{\mu} X^{J}\right), \quad \partial_{[\mu} \mathcal{A}_{\nu]}=0
$$

Furthermore we find that the fields $Y_{i j}^{I}, \hat{B}_{i j}$ and the curvatures associated with the U(1), SU(2) and dilatational gauge fields vanish, whereas the field $D$ becomes proportional to $\mathcal{F}_{a b}^{-} T^{i j a b}$, which will vanish as we shall prove below. Then by using the definition of $G_{\mu \nu I}^{-}$, the second relation of (17) and the homogeneity property (6), the field $T_{a b}^{i j}$ can be written as follows,

$$
T_{a b}^{i j}=2 i \varepsilon^{i j} \mathrm{e}^{\mathcal{K}}\left[F_{I} F_{a b}^{-I}-X^{I} G_{a b I}^{-}\right] .
$$

This happens to be the same relation that one derives by solving the field equations for the field $T^{a b i j}$ (in the presence of a fixed chiral background), which incidentally takes the form $\mathcal{F}_{a b}=0$. Note, however, that the right-hand side of this equation also depends on $T^{a b i j}$. We also note the relation

$$
-\frac{1}{4} \varepsilon_{i j} T_{01}^{i j}+i \mathcal{F}_{01}^{+}=\frac{1}{r^{2}} \mathrm{e}^{-2 f(r)} \mathrm{e}^{\mathcal{K} / 2} Z .
$$

In addition, we find that the spacetime geometry has to satisfy

$$
C_{a b}^{-c d}=-\frac{1}{24} i \varepsilon_{i j} T^{e f i j} \mathcal{F}_{e f}^{-}\left(\delta_{a b}^{c d}-\frac{1}{2} \varepsilon_{a b}^{c d}\right)+\frac{1}{8} i \varepsilon_{i j}\left[T_{a b}^{i j} \mathcal{F}^{-c d}+T^{c d i j} \mathcal{F}_{a b}^{-}\right],
$$

where the tensor $C_{a b}^{-c d}$, which is antiselfdual in both pairs of indices, is just the traceless part of the curvature of the spin connection field $\omega_{\mu}^{a b}$ in the presence of the dilatational gauge field $b_{\mu}$. Once we fix the scale with respect to dilatations, this tensor becomes the (antiselfdual part of the) Weyl tensor. As it turns out, in a spherically symmetric geometry, $C_{a b}^{-c d}$ can only be zero, so that $\mathcal{F}_{a b}^{-} T^{c d i j}=0$. This proves that the field $D$ is zero indeed.

At this point two more features are relevant. First of all, as alluded to in the introduction, in order to have a consistent action one needs two compensating supermultiplets [10]. One of them 
is a vector multiplet, which we have already taken into account in our analysis given above and which provides the graviphoton. For the second compensating supermultiplet there are various options, but they all share the feature that the corresponding spinor does not transform into a Lorentz vector that takes its values in the chiral $u(2)$ algebra or into a (anti)selfdual Lorentz tensor. The presence of the second compensator is also crucial to make the vanishing value of $D$ compatible with the equations of motion. We will not discuss this rather subtle issue in any detail here, but note that the vanishing of the supersymmetry variation of the spinor belonging to the second compensating multiplet yields two more conditions,

$$
\mathcal{F}_{a b}^{-}=0, \quad \mathcal{A}_{\mu}+A_{\mu}=0 .
$$

Then, it follows from (16) and (17) that $\mathcal{D}_{\mu}\left(\mathrm{e}^{\mathcal{K} / 2} X^{I}\right)=\mathcal{D}_{\mu}\left(\mathrm{e}^{\mathcal{K} / 2} F_{I}\right)=\mathcal{D}_{\mu}\left(\mathrm{e}^{w \mathcal{K} / 2} \hat{A}\right)=\mathcal{F}_{a b}^{-I}=$ $\hat{F}_{a b}^{-}=0$. From (21), on the other hand, we obtain $\frac{1}{4} \varepsilon_{i j} T_{01}^{i j}=\frac{1}{4} i \varepsilon_{i j} T_{23}^{i j}=-r^{-2} \mathrm{e}^{-2 f(r)} \mathrm{e}^{\mathcal{K} / 2} Z$.

Secondly, since the supersymmetry transformations of all fermionic quantities should vanish in the supersymmetric background, also derivatives of spinors should have a zero variation. Often this does not lead to new results, but in the case at hand, it does. Namely, requiring that $D_{\mu} \zeta_{i}$ vanishes under $Q$-supersymmetry up to the uniform $S$-transformation that we mentioned before, yields the following equation for the gauge field $f_{\mu}^{a}$ associated with special conformal boosts:

$$
f_{\mu a}-\frac{1}{2} \mathcal{D}_{\mu} \mathcal{D}_{a} \mathcal{K}-\frac{1}{4} \mathcal{D}_{\mu} \mathcal{K} \mathcal{D}_{a} \mathcal{K}+\frac{1}{8}\left(\mathcal{D}_{b} \mathcal{K}\right)^{2} e_{\mu a}=0
$$

Here, it is important to recall that $f_{\mu}^{a}$ is not an independent, but a composite gauge field [10], given by $f_{\mu}^{a}=\frac{1}{2} R_{\mu}^{a}-\frac{1}{4}\left(D+\frac{1}{3} R\right) e_{\mu}^{a}+\frac{1}{16} T^{a b i j} T_{\mu b i j}$.

The superconformal theory described above is gauge equivalent to a Poincaré supergravity theory. A Poincaré frame is chosen by expressing the results in terms of scale-invariant fields, such as the rescaled vierbein $e_{\mu}^{a} \exp [-\mathcal{K} / 2]$, or, equivalently, by choosing a gauge in which $\mathcal{K}$ is constant and $b_{\mu}=0$. In this frame $f_{\mu a}=0$, so that the Ricci tensor associated with the spacetime line element (11) equals $R_{\mu}^{a}=-\frac{1}{8} T^{a b i j} T_{\mu b i j}$. Furthermore, using (23), we find from (18) that $\mathcal{D}_{\mu} T^{a b i j}=0$. Now recall that the $\mathrm{U}(1)$-connection $A_{\mu}$ is a flat connection in the $N=2$ supersymmetric background. Picking a gauge in which $A_{\mu}=0$ and using the fact that the spacetime line element has spherical symmetry, we conclude that $X^{I}, F_{I}, \hat{A}, T^{a b i j}$ and therefore $Z$ are all constant. From this it follows that $f(r)+\ln r$ is constant as well. Thus, the only nonvanishing components (with tangent-space indices) of the Ricci tensor are $R_{00}=R_{22}=R_{33}=-R_{11}=-\frac{1}{16}\left|T^{01 i j} \varepsilon_{i j}\right|^{2}$, while the Weyl tensor is zero. These requirements restrict the line element (up to diffeomorphisms) to be of the Bertotti-Robinson type:

$$
e^{2 g(r)}=e^{-2 f(r)}=\frac{1}{16}\left|T^{01 i j} \varepsilon_{i j}\right|^{2} r^{2}=\mathrm{e}^{-\mathcal{K}} \frac{r^{2}}{|Z|^{2}},
$$

where we note the relation

$$
T_{01 i j}=-2 \varepsilon_{i j} \mathrm{e}^{-\mathcal{K} / 2} Z^{-1} .
$$

Hence we have proven that there are two fully supersymmetric solutions for off-shell multiplets with an arbitrary chiral background field that are static and spherically symmetric, namely Bertotti-Robinson and, when $T^{a b i j}=0$, flat Minkowski spacetime. 
Now we write down the Lagrangian of the vector multiplets in the chiral background [12],

$$
\begin{aligned}
8 \pi \mathcal{L}= & -\frac{1}{2} \mathrm{e}^{-\mathcal{K}} R+\left[i \mathcal{D}^{\mu} F_{I} \mathcal{D}_{\mu} \bar{X}^{I}-\frac{1}{8} i F_{I J} Y_{i j}^{I} Y^{J i j}-\frac{1}{4} i \hat{B}_{i j} F_{\hat{A} I} Y^{I i j}\right. \\
& +\frac{1}{4} i F_{I J}\left(F_{a b}^{-I}-\frac{1}{4} \bar{X}^{I} T_{a b}^{i j} \varepsilon_{i j}\right)\left(F_{a b}^{-J}-\frac{1}{4} \bar{X}^{J} T_{a b}^{i j} \varepsilon_{i j}\right) \\
& -\frac{1}{8} i F_{I}\left(F_{a b}^{+I}-\frac{1}{4} X^{I} T_{a b i j} \varepsilon^{i j}\right) T_{a b i j} \varepsilon^{i j}+\frac{1}{2} i \hat{F}_{a b}^{-} F_{\hat{A} I}\left(F_{a b}^{-I}-\frac{1}{4} \bar{X}^{I} T_{a b}^{i j} \varepsilon_{i j}\right) \\
& \left.+\frac{1}{2} i F_{\hat{A}} \hat{C}-\frac{1}{8} i F_{\hat{A} \hat{A}}\left(\varepsilon^{i k} \varepsilon^{j l} \hat{B}_{i j} \hat{B}_{k l}-2 \hat{F}_{a b}^{-} \hat{F}_{a b}^{-}\right)-\frac{1}{32} i F\left(T_{a b i j} \varepsilon^{i j}\right)^{2}+\text { h.c. }\right] .
\end{aligned}
$$

Here we have taken into account the effect from the second compensator in that we changed the coefficient of the first term by a factor 3 and we suppressed the terms linear in the field $D$. Now we identify the chiral background superfield with the superfield $\left[\varepsilon_{i j} W^{a b i j}\right]^{2}$, so that $\hat{A}=\left(\varepsilon_{i j} T^{a b i j}\right)^{2}$. It is tedious but straightforward to determine the higher components of the chiral background 111. Here we need only the bosonic part of $\hat{C}$, which is given by $\hat{C}=$ $-8 \varepsilon_{i j} T^{a b i j}\left\{\mathcal{D}_{a}, \mathcal{D}^{c}\right\} T_{c b k l} \varepsilon^{k l}+16 \varepsilon_{i j} T^{a b i j} f_{a}^{c} T_{c b k l} \varepsilon^{k l}+64 \mathcal{R}(M)_{c d}^{-a b} \mathcal{R}(M)_{c d}^{-a b}+32 R(\mathcal{V})_{a b l}^{-k} R(\mathcal{V})_{a b k}^{-l}$, where $\mathcal{R}(M)_{a b}{ }^{c d}=R_{a b}{ }^{c d}-4 \delta_{[a}^{[c} f_{b]}{ }^{d]}+\frac{1}{16}\left(T^{i j c d} T_{i j a b}+T_{a b}^{i j} T_{i j}^{c d}\right)$. We note that, after going into the Poincaré frame, the Lagrangian contains $R^{2}$-terms, but no terms involving derivatives of the Rieman tensor.

The solution we have constructed describes the near-horizon limit of an extremal black-hole solution in the presence of $R^{2}$-terms in the effective Lagrangian (27). In order to compute the macroscopic entropy associated with these solutions, we will use Wald's formalism [7] and view the entropy as a Noether charge associated with an isometry generated by the static Killing vector field of the Bertotti-Robinson geometry. Even though Wald's derivation of the First Law of black-hole mechanics holds primarily for nonextremal black holes, we will in the following assume that the corresponding entropy formula can be extrapolated to the case of extremal black holes. Since our findings are consistent with electric-magnetic duality, we are confident that this extrapolation makes perfect sense. For a class of Lagrangians of the form (27) taken in the Poincaré frame, the entropy is, according to Wald's proposal [7], given by

$$
\mathcal{S}=\frac{1}{16} \oint_{S^{2}} \epsilon_{a b} \epsilon_{c d} \frac{\delta(8 \pi \mathcal{L})}{\delta R_{a b c d}}
$$

where the indices of the Levi-Civita symbol $\epsilon_{a b}$ run over $a, b=0,1$ and where the integral is over a spatial cross-section of the Killing horizon, which in this case is just $S^{2}$. Using (1), the integral thus yields an overall factor $4 \pi r^{2} \exp [2 f(r)]$. Taking the derivative of the Lagrangian (27) with respect to the Riemann tensor in the $(r, t)$-subspace leads to various terms. Some of them cancel in the supersymmetric background and we are left with the variation of the first term in (27) and with the variation of the term in $\hat{C}$ proportional to $f T^{2}$. The result takes the form

$$
\mathcal{S}=\pi\left[|Z|^{2}-256 \operatorname{Im}\left[F_{\hat{A}}\left(X^{I}, \hat{A}\right)\right]\right], \quad \text { with } \quad \hat{A}=-64 \bar{Z}^{-2} \mathrm{e}^{-\mathcal{K}},
$$

where the $X^{I}$ and therefore $Z$ are specified by (11) in terms of the charges carried by the extremal black hole. The first term in the entropy formula coincides with the Bekenstein-Hawking area contribution. It is important to note that the second term involves only the first derivative of the function $F$ with respect to the background field, which, unlike higher derivatives, transforms as a function under symplectic transformations [12]. Thus our result is consistent with electricmagnetic duality. 
We conclude this letter with the explicit computation of the macroscopic entropy of a certain class of extremal black-hole solutions occuring in string effective field theories arising from Calabi-Yau compactifications, and we demonstrate its perfect agreement with the counting of microstates for this class of black holes performed in [1, 2]. We refer to an upcoming publication 131 in which we will discuss further examples, such as heterotic black holes, as well as corrections to the entropy formulae of [1, 2] due to the inclusion of contributions coming from terms in the Lagrangian proportional to $T^{2(g-1)} R^{2}$ with constant coupling functions [14, 15, 16], and also corrections steming from certain nonperturbative effects [16].

It will be convenient $\| \mathbb{A}$ to use rescaled variables $Y^{I}=\mathrm{e}^{\mathcal{K} / 2} \bar{Z} X^{I}$ and $\Upsilon=\mathrm{e}^{\mathcal{K}} \bar{Z}^{2} \hat{A}$. Using the homogeneity property of $F$, it then follows that $Z=\bar{Z}^{-1}\left(p^{I} F_{I}(Y, \Upsilon)-q_{I} Y^{I}\right)$ and the stabilization equations (11) read $Y^{I}-\bar{Y}^{I}=i p^{I}$ and $F_{I}(Y, \Upsilon)-\bar{F}_{I}(\bar{Y}, \bar{\Upsilon})=i q_{I}$. Furthermore the entropy formula (29) takes the form $\mathcal{S}=\pi\left[Z \bar{Z}-256 \operatorname{Im} F_{\Upsilon}(Y, \Upsilon)\right]$ with $\Upsilon=-64$. Let us now consider type-IIA string theory compactified on a Calabi-Yau threefold, in the limit where the volume of the Calabi-Yau threefold is taken to be large. For the associated homogenous function $F(Y, \Upsilon)$ we take (with $I=0, \ldots, n$ and $A=1, \ldots, n$ )

$$
F(Y, \Upsilon)=\frac{D_{A B C} Y^{A} Y^{B} Y^{C}}{Y^{0}}+d_{A} \frac{Y^{A}}{Y^{0}} \Upsilon, \quad D_{A B C}=-\frac{1}{6} C_{A B C}, \quad d_{A}=-\frac{1}{24} \frac{1}{64} c_{2 A}
$$

where the coefficients $C_{A B C}$ denote the intersection numbers of the four-cycles of the CalabiYau threefold, whereas the coefficients $c_{2 A}$ denote its second Chern-class numbers [14]. The Lagrangian (27) associated with this homogenous function thus contains a term proportional to $c_{2 A} z^{A} R^{2}$, where $z^{A}=Y^{A} / Y^{0}$. In the following we will consider black holes with $p^{0}=0$. For real $\Upsilon$ the solution of the stabilization equations equals

$$
\begin{aligned}
& Y^{0}=\bar{Y}^{0}, \quad\left(Y^{0}\right)^{2}=\frac{D_{A B C} p^{A} p^{B} p^{C}-4 d_{A} p^{A} \Upsilon}{4\left(q_{0}+\frac{1}{12} D^{A B} q_{A} q_{B}\right)}, \\
& Y^{A}=\frac{1}{6} Y^{0} D^{A B} q_{B}+\frac{1}{2} i p^{A}, \quad D_{A B}=D_{A B C} p^{C}, \quad D_{A B} D^{B C}=\delta_{A}^{C} .
\end{aligned}
$$

Switching off the background field $\Upsilon$ yields the solution presented in [17]. Furthermore we find

$$
Z \bar{Z}=-4 Y^{0}\left(q_{0}+\frac{1}{12} D^{A B} q_{A} q_{B}\right)-2 \frac{d_{A} p^{A}}{Y^{0}} \Upsilon .
$$

Inserting this into the entropy formula, and using $\Upsilon=-64$, yields

$$
\mathcal{S}=-4 \pi Y^{0}\left(q_{0}+\frac{1}{12} D^{A B} q_{A} q_{B}\right)
$$

Since the coefficients $D_{A B C}$ are negative, we take $\hat{q}_{0}=q_{0}+\frac{1}{12} D^{A B} q_{A} q_{B}<0$ and $p^{A}>0$, so that $\left(Y^{0}\right)^{2}>0$. Choosing $Y^{0}$ positive as well, so that the moduli $z^{A}$ live in the upper half-plane $\operatorname{Im} z^{A}>0$, we thus see that the presence of the particular $R^{2}$-term associated with (30) in the Lagrangian (27) results in a correction to the macroscopic entropy which is in exact agreement with the microscopic entropy formula computed in [1, 2]:

$$
\mathcal{S}_{\text {micro }}=2 \pi \sqrt{\frac{1}{6}\left|\hat{q}_{0}\right|\left(C_{A B C} p^{A} p^{B} p^{C}+c_{2 A} p^{A}\right)} .
$$

\section{Acknowledgements}

We thank K. Behrndt, G. Horowitz, T. Jacobson, R. Kallosh, B. Kleijn, D. Lüst, S. Massar and R. Myers for valuable discussions. The work of G.L.C. was supported in part by the European Commission TMR programme ERBFMRX-CT96-0045. 


\section{References}

[1] J. M. Maldacena, A. Strominger and E. Witten, J. High Energy Phys. 12 (1997) 2, hep-th/9711053.

[2] C. Vafa, Adv. Theor. Math. Phys. 2 (1998) 207, hep-th/9711067.

[3] S. Ferrara, R. Kallosh and A. Strominger, Phys. Rev. D52 (1995) 5412, hep-th/9508072;

S. Ferrara and R. Kallosh, Phys. Rev. D54 (1996) 1514, hep-th/9602136; Phys. Rev. D54 (1996) 1525, hep-th/9603090.

[4] K. Behrndt, G. L. Cardoso, B. de Wit, R. Kallosh, D. Lüst and T. Mohaupt, Nucl. Phys. B488 (1997) 236, hep-th/9610105.

[5] A. Strominger and C. Vafa, Phys. Lett. B379 (1996) 99, hep-th/9601029.

[6] K. Behrndt, G. L. Cardoso, B. de Wit, D. Lüst, T. Mohaupt and W. A. Sabra, Phys. Lett. B429 (1998) 289, hep-th/9801081.

[7] R. M. Wald, Phys. Rev. D48 (1993) 3427, gr-qc/9307038; T. Jacobson, G. Kang and R. C. Myers, Phys. Rev. D49 (1994) 6587, gr-qc/9312023; V. Iyer and R. M. Wald, Phys. Rev. D50 (1994) 846, gr-qc/9403028; T. Jacobson, G. Kang and R. C. Myers, Black-Hole Entropy in Higher Curvature Gravity, gr-qc/9502009.

[8] G. Gibbons, in Supersymmetry, Supergravity and Related Topics, eds. F. del Aguila, J. de Azcárraga and L. Ibánẽz, World Scientific (1985) 147.

[9] G. Moore, Attractors and Arithmetic, hep-th/9807056; Arithmetic and Attractors, hep-th/9807087.

[10] B. de Wit, J. W. van Holten and A. Van Proeyen, Nucl. Phys. B167 (1980) 186, Nucl. Phys. B184 (1981) 77; B. de Wit and A. Van Proeyen, Nucl. Phys. B245 (1984) 89; B. de Wit, P. G. Lauwers and A. Van Proeyen, Nucl. Phys. B255 (1985) 569.

[11] E. Bergshoeff, M. de Roo and B. de Wit, Nucl. Phys. B182 (1981) 173.

[12] B. de Wit, Nucl. Phys. (Proc. Suppl.) B49 (1996) 191, hep-th/9602060; Fortsch. Phys. 44 (1996) 529, hep-th/9603191.

[13] G. L. Cardoso, B. de Wit and T. Mohaupt, to appear.

[14] M. Bershadsky, S. Cecotti, H. Ooguri and C. Vafa, Nucl. Phys. B405 (1993) 279, hep-th/9302103; Comm. Math. Phys. 165 (1994) 311, hep-th/9309140.

[15] M. Mariño and G. Moore, Counting higher genus curves in a Calabi-Yau manifold, hep-th/9808131.

[16] R. Gopakumar and C. Vafa, M-Theory and Topological Strings-I, hep-th/9809187.

[17] M. Shmakova, Phys. Rev. D56 (1997) 540, hep-th/9612076. 